\title{
THE HEATING PATTERN OF THE MICROWAVE DEHYDRATOR FOR TREATING PETROLEUM CRUDE OILS
}

\author{
Souleyman A. Issaka ${ }^{1}$, Abdurahman H. Nour ${ }^{2}$, Rosli Mohd Yunus ${ }^{3}$, Azhari, H.Nour ${ }^{4}$ \\ ${ }^{1,2,3,4}$ Faculty of Chemical and Natural Resources Engineering, University Malaysia Pahang
}

\begin{abstract}
The effect of microwave dehydrator (modified domestic microwave oven) different types of petroleum oils were investigated in this paper, since microwave heats material selectively and volumetrically in relatively short time, and since its application in treating petroleum emulsion is tremendously recommended, thereof, it is the aim of this paper to investigate the microwave induced heating capacities of the individual components of the petroleum emulsion mixture. Three types of crude petroleum oils and water samples were put into test, from the result of this study, the temperature profile was observed to proceed as follow, at the first 20 seconds, all samples had similar temperature profiles in the range $\left(50-60^{\circ} \mathrm{C}\right)$, however, beyond 20 seconds there was a clear distinct trend for every sample, in the interval from 20 seconds to 110 seconds, water sample had the curve with the highest temperature profile followed by UAE crude oil, then Saudi Arabia oil. Beyond 110 seconds, Kuwait crude oil had the maximum temperature profile continued sharply without reaching any plateau, followed by UAE then Saudi Arabia crude oil. The volumetric rate of heat generation also found to be in such a way that water sample had the maximum numerical value in the range $\left(1-1.2 \mathrm{cal} / \mathrm{s.cm}{ }^{3}\right)$ at the beginning then decays as the heating time goes on, followed by that of Kuwait oil (0.8-1 cal/s.cm $\left.{ }^{3}\right)$, then Saudi Arabia crude oil (0.6-0.8 $\left.\mathrm{cal} / \mathrm{s} . \mathrm{cm}^{3}\right)$. Furthermore water also found to have the highest dielectric loss ranged between (7-8) and lowest wavelength in the range (1.5-2) followed by all the oil samples which had constant and identical dielectric properties in the range (0-0.5) and wavelength of 8 $\mathrm{Cm}$. from the experimental results of this research, one can conclude that although both water and crude oils can interact effectively with microwave radiation yet the mechanism may not be the same since oils were shown to have very low dielectric loss. Another observation is different crude oils have different heating pattern, there this had to be into consideration when studying crude emulsion or refining.
\end{abstract}

Keywords: microwave dehydrator, heating petroleum oils, dielectric of petroleum, and dielectric of water.

\section{INTRODUCTION}

This article shall investigate the effects of microwave heating on some petroleum crude oils, the purpose of heating the pure petroleum components is to gain some understanding on how the individual phases of the of petroleum emulsion are heated, thus the profile and dielectric properties of several three crude oils and water phases shall be investigated.

The dramatic increase in the prices of energy these days had catalyzed the desire to find new energy efficient ways of industrial process (D. A. Jones et al., 2002), one of such ways is the introduction of microwave in the processing industry ( $\mathrm{T}$. J. Appleton et al., 2005), this paper shall investigate the heating capacity of the domestic microwave on the individual components that form the petroleum based emulsions. That is because it has proven to be more efficient and safe from both economic and environmental perspective (D. A. Jones et al., 2002), (Su shiung lam et al., 2012). Microwave heats materials volumetrically unlike the conventional heating, in microwave heating, different materials get heated up differently depending on their capacity to absorb the wave and convert them to heat, hence only materials that process dielectric properties can absorb microwave energy (J. P. Robinson et al., 2008).

Fundamentally, microwave is a term used to designate the thermal radiation waves falling in the range between $300 \mathrm{MHz}$ to $300 \mathrm{GHz}$ (J. A. Menendez et al., 2010). Technically speaking; most of the domestic oven as well as industrial microwaves devises function at fixed frequency of $2.45 \mathrm{GHz}$ and various power levels. The usual wavelength for domestic microwaves is $12.2 \mathrm{~cm}$ and the energy is $1.02 * 10^{\wedge}-5 \mathrm{eV}$ (D. A. Jones et al., 2002),(Adrian Porch et al., 2012) .

Quan $\mathrm{Bu}$ and friends have used the microwave energy to produce usable hydrocarbons from Saw dust (Quan Bu et al., 2013). Microwave heats materials selectively and volumetrically by inducing the orientation of charges as well as dipoles at short processing time than the corresponding conventional heating without affecting the recovered oil quality (Rocio Perez-Paez et al., 2008). Thus far microwave energy is well used in certain areas of research including, Analytical chemistry, Microwave organic synthesis and centering of ceramics (Vasily et al., 2012), indeed microwave also reported to decrease the temperature of the chemical 
reaction (Vasily et al., 2012). Microwave energy may also be applied in petroleum distillation especially the heavy polar residues (Adrian Proch et al., 2012).

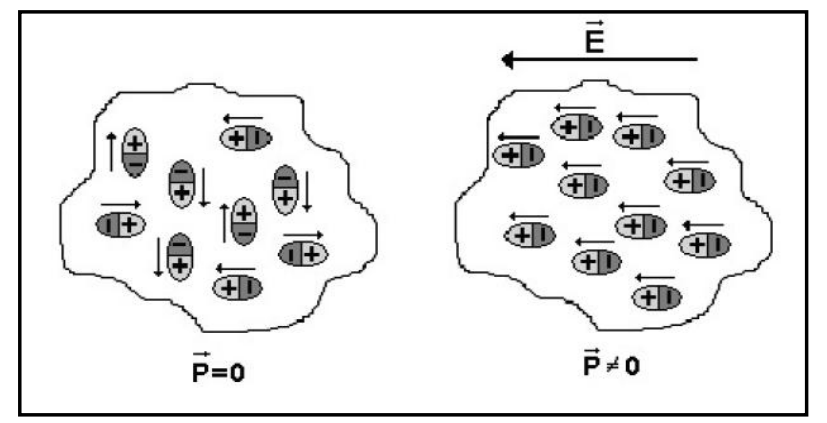

Fig 1: Dipole orientation under the microwave field (Rocio Perez-paez et al., 2008)

Generally the electromagnetic power in converted to energy within the absorbing materials, hence the power density per unit volume is given mathematically by (H. Shang et al., 2006)

$$
\mathrm{P}_{\mathrm{d}}=2 \pi \mathrm{f} \varepsilon_{0} \varepsilon^{\prime \prime}|\mathrm{E}|^{2}
$$

Where:

$\mathrm{f}$ : Frequency $(\mathrm{Hz})$

$\varepsilon_{0}:$ Permittivity of the free space $\left(8.85 * 10^{\wedge}-12\right)$

$\varepsilon^{\prime \prime}$ : Dielectric loss

E: magnitude of the electric field

Most of this absorbed energy is used to heat up the sample by increasing its temperature; hence the relation between the power absorption and rate of temperature rise is given by

$$
\frac{\Delta \mathrm{T}}{\Delta \mathrm{t}}=\frac{2 \pi \mathrm{f} \varepsilon_{0} \varepsilon^{\prime \prime}|\mathrm{E}|^{2}}{\rho \mathrm{C}_{\mathrm{p}}}
$$

Where $\frac{\Delta T}{\Delta t}$ is the rate of temperature increase within the irradiated sample as the irradiation goes on.

From the previous equation (1), it is evident that, the temperature rise within the material is directly proportional with the square of the electric field strength, therefore in designing the cavity, a profound definition of electric field is a must (single mode and multiple mode) (H. Shang et al.,2006). One of the most important mechanism with which microwave interacts with material is the so called polarization effect. The polarization is the mechanism of alignment reorientation of the dipole or free charges of the system as electromagnetic wave penetrates through, as depicted in figure 1 (Rocio Perez-paez et al., 2008). Dipole is the state of bonding structure wherein the atoms around molecules are unevenly balanced $(\mathrm{N}$. Bjorndalen et al., 2004). The heat generation process at the dipole level would happen in such a way that, the dipoles try to align and realign themselves (Oscilate) continuously with the alternating electromagnetic field, thus molecules switch or flip approximately 2.5 billion time/second when frequency range is $2.45 \mathrm{GHz}$ (domestic microwave oven), this huge vibration would cause frictions among particles in the medium that would generate energy in the forms of heat (T. J. Appleton et al., 2005). The rate of temperature increase and temperature raise becomes higher as the microwave power level increases (Zhifeng $\mathrm{Hu}$ et al., 2012, normal domestic ovens mostly poses five levels of power, from very low to very high. Before designing microwave cavity for industrial or pilot application, a good knowledge of dielectric properties is crucial, however microwave also proved to reduce energy consumption, increase process yield as well as abate some environmental issues (D. A. Jones et al., 2002). The strength of the electromagnetic field within the materials depends upon several parameters including, the power level of microwave, geometry of the sample holder as well as the portion of the incident wave that is absorbed by the material. The absorbed portion of the wave is in turn a very strong function of dielectric characteristics of material, the dielectric properties consists of two major parts, first of which is dielectric constant representing the ability of materials to obstruct or store the electromagnetic energy as it passes through, while the second phenomena is the ability of materials to convert that stored energy to heat. Both dielectric constant and dielectric loss are functions of frequency, temperature, and types of materials ( $\mathrm{J}$. P. Robinson et al., 2012). Large diameter applicator can increase the surface area and improve wave absorption, but beyond a certain diameter called critical diameter, the effect would reverse and the opposite trend would take place (J. Zhu et al., 2007).

So many researchers have suggested the use of microwave as an alternative method to treat petroleum emulsion. Mingfu Huang and friends have tried the use of microwave in deacidification of distillate crude oil, however from their results they have suggested several advantages of microwave over the conventional heating, microwave was reported to be highly efficient, less processing time as well as environmental friendly (Mingfu Huang et al., 2006).

Other researchers used microwave to treat crude oil contaminated soil at elevated temperature, however they used Carbone fibers as absorbers to increase the microwave properties of the soil (Li Dawei et al., 2009). Elsewhere, microwave was applied to treat soil contaminated with crude oil, paraffin oil and asphaltene (N. Bjorndalen et al., 2004).

In one article, it had reported that the presence of water in the soil sample helped the sample to attain elevated dielectric properties at low temperature below $100^{\circ} \mathrm{C}$ (J. P. Robinson et al., 2012).

Elsewhere the potential of use microwave energy in the refinement of heavy fraction of the petroleum oil was 
investigated by determining the complex permittivity of the heavy oil as function frequencies (Adrian porch et al., 2012).

Microwave was suggested as a mean to heat petroleum in order to upgrade crude oil, remove heteroatom, as well as facilitate the pumping from underground (viscosity reduction) (Sateesh Mutyala et al.,2010). Microwave also used to pyrolyse the waste automotive engine oil to produce some useful fuels (Su shiung lam et al., 2012).

Massive amount of budget have been allocated as research fund to implement microwave technology in the crude oil processing application in last three decades, however, most of the effort done thus far have paved the way to ask more questions than it provide solutions (Sateesh Mutyala et al., 2010).

Since microwave is currently recommended as very convenient way of processing, therefore it is the aim of this research to investigate effect of microwave radiation on heating properties of various types of liquids, including water and several crude oils. That is to get some ideas on how the individual materials of emulsions respond to microwave energy.

\section{MATERIALS AND METHODS}

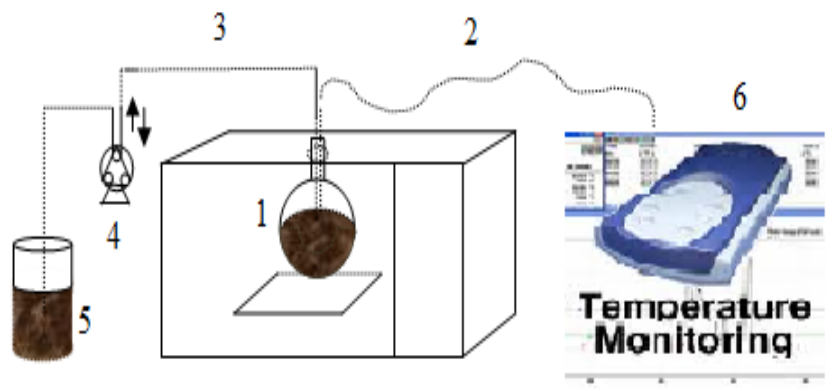

Fig 2: Microwave dehydrator (MD)

Figure 2 is a schematic representation of the apparatus used to carry out this experimental research, 1) sample holder (Round bottom flask) inside the microwave oven, 2) thermocouple for sensing the temperature, 3) hose to move the sample (emulsion) in and out, 4) peristaltic pump, 5) sample tank, 6) Pico data Logger for temperature monitoring.

The system consists of domestic microwave oven, with maximum power output of 800 watt, and $2450 \mathrm{MHz}$ frequencies, in this study, the operation frequencies were set at 800 watt, a container of $250 \mathrm{ml}$ round bottomed flask was used, a thermo couple was used to record the temperature through a Pico data logger connected to computer.

\subsection{Equation used for Data Interpretation}

Equation 3 is used to calculate the heat generation of the treated samples, after the convective and radiation terms were eliminated from the general heat transfer equation.

$$
q_{M W}=\rho C_{p} \frac{d T}{d t}
$$

Where:

$\mathrm{q}_{\mathrm{mw}}$ : Volumetric rate of heat generation $(\mathrm{Cal} / \mathrm{s} . \mathrm{cm} 3)$

$\rho$ and $C_{p}$ : are the density and heat capacity of materials $(\mathrm{g} / \mathrm{C} 3)$ and $\left(\mathrm{Cal} / \mathrm{g} .^{\circ} \mathrm{C}\right)$

$\mathrm{dT} / \mathrm{dt}$ : The rate of temperature increase $\left({ }^{\circ} \mathrm{C} / \mathrm{S}\right)$

The temperature data are collected using the Picodata Loger device which is connected to PC,

The dielectric constant and dielectric loss of water used for the model in this study was given by wolf, (Wolf. 1986)

$$
\begin{aligned}
& \varepsilon_{r^{\prime} w}^{\prime}=85.215-0.33583 T \\
& \varepsilon_{r^{\prime} w}=320.685 T^{-1.0268}
\end{aligned}
$$

\section{Where $\varepsilon^{\prime}$ : Dielectric constant of water $\varepsilon^{\prime \prime}:$ Dielectric loss of water}

Van Hippel. (1954), proposed equation for dielectric properties of various petroleum oils, in this regard dielectric constant and loss tangent of crude oil for this study calculated from the equation below:

$$
\varepsilon_{r^{\prime} o}^{\prime}=2.24-0.000727 T
$$

\subsection{Measurement of Penetration Depth and Wavelength within the Heated Sample}

Gallawa (1989) reported that, there is a direct relationship between the frequency and the wavelength. There is also a relationship between the frequency and the degree, or speed of heating, as well as the depth of penetration. The wavelength (which is the distance between positive peaks or negative peaks of two adjacent waves) is equal to the speed of light divided by the frequency. For practical purposes, the speed of light does not change. Therefore, the only two variables in equation are the frequency and the wavelength, their relationship as follows:

$$
\lambda_{m}=\frac{c}{f}
$$




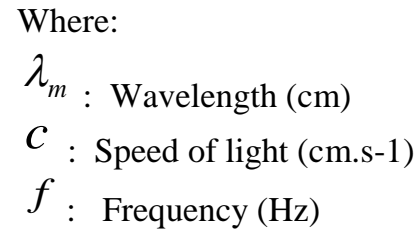

The above relation indicates, the higher the frequency, the shorter the wavelength, and vice versa (low frequency, corresponds to longer the wavelength. (Tanmay (2004) related the microwave penetration depth $D_{p}$ and wavelength of radiation in the medium $\lambda_{m}$ to dielectric constant $\varepsilon^{\prime}{ }_{r}$ and dielectric loss (loss factor) $\varepsilon^{\prime \prime} r$ in the following manner.

$$
D_{p}=\frac{c}{\sqrt{2} * \pi * f\left[\varepsilon_{r}^{\prime}\left(\sqrt{1+\left(\frac{\varepsilon_{r}^{\prime \prime}}{\varepsilon_{r}^{\prime}}\right)^{2}}-1\right)\right]^{1 / 2}}
$$

And

$$
\lambda_{m}=\frac{c \sqrt{2}}{f\left[\varepsilon^{\prime} r\left(\sqrt{1+\left(\frac{\varepsilon^{\prime \prime}{ }_{r}}{\varepsilon_{r}^{\prime}}\right)^{2}}+1\right)\right]^{1 / 2}}
$$

The two most common techniques for dielectric properties measurements are the resonant cavity, or cavity perturbation method, and the transmission and reflection method, however, the current researchers used the existing correlation to relate wavelength to the dielectric properties as given in equation (9).

\section{RESULTS AND DISCUSSION}

Three different samples were used, namely; two petroleum oils and one water, In this study the microwave power level was fixed at the maximum of 800 watt, the irradiation time was recorded from zero to 3.5 minutes with one minute increment, then interpreted as shown in the graphs, actually each point in the $\mathrm{X}$-axis of the graph is average of 10 points in the original data, the variables investigated were the temperature profile, volumetric heat generation and dielectric properties.

\subsection{The Temperature Profiles of the Individual}

\section{Components}

The heating properties of the pure components (water and crude oils) were carried out and their results were plotted in the subsequent graphs.

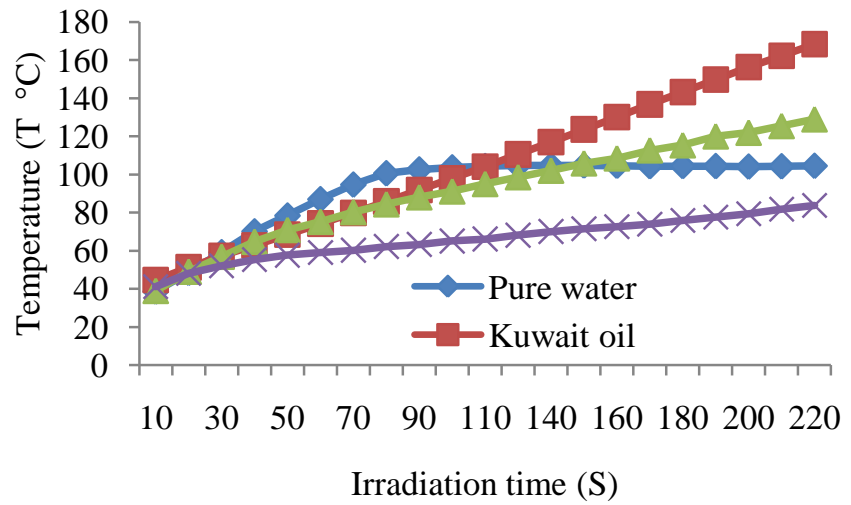

Fig 3: Heating of pure water and pure oils at 800 Watt for 3.5 minutes

Figure 3 represents the experimental data of the pure Raw materials (water, and three different crude oils), as it is clear from the Figure 3, in all cases initially within the first 20 seconds; all components had similar temperature profile of more or less $50{ }^{\circ} \mathrm{C}$ and gradually the temperature trend of each component had taken different paths, thus water had the path with the maximum values of temperature compared to the others. within the interval from 20 seconds until 90 seconds, that was in accordance with the theory which consider water as more polar than hydrocarbons (oils) and supposed to absorb and generate more energy from the incident electromagnetic wave that could be evidenced by the increase of the temperature (Alvin et al.,2009), (Vasily A. Bolotov et al., 2012).

however at 90 seconds, the temperature of water had reached a plateau at around $100{ }^{\circ} \mathrm{C}$ and remained constant till the end of the irradiation period of 210 seconds, that plateau could be attributed to the fact that the dielectric properties (would be discussed latter on) which responsible of converting the electromagnetic wave into energy, note that an increase in irradiation time will increase the sample temperature, another possible cause of plateau in the temperature of water may be due to physical properties such as boiling point, heat capacity and density (F. Kormin et al., 2013). Beside that for Kuwait and UAE oils had to some extend equivalent trend evidenced by the overlap on their two curves within the irradiation period between $20 \mathrm{~s}$ to $80 \mathrm{~s}$, they also appeared to be the components with the second highest temperature path in this particular interval, but after that interval or after $80 \mathrm{~s}$ was elapsed; the path of the Kuwait oil had increased even more and intersect with water path at $105{ }^{\circ} \mathrm{C}$, after that it exceeded the water path, and continued sharply to $170{ }^{\circ} \mathrm{C}$ at the end of the irradiation time, this in a direct disagreement with the electromagnetic theory which strongly relates microwave heating with polarity and conductivity, however authors attributed this strange thermal behavior of the Kuwait crude oil under the microwave heating to the existence of some ionic elements or fatty acids within the crude oils, since microwave 
heating of materials is a strong function of temperature and thus materials with low dielectric properties including insulators tend to absorb and couple more effectively with microwave energy as temperature increase (T. J. Appleton et al., 2005).

UAE oil had taken another path with a bit lower temperature than Kuwait oil and intersected with water curve at $145 \mathrm{~s}$, then exceeded the water path and reached around $120{ }^{\circ} \mathrm{C}$ at the end of the irradiation time, finally Saudi Arabian crude oil was observed to have the lowest thermal properties with a maximum temperature of around $80{ }^{\circ} \mathrm{C}$, , this is somehow in accordance with the electromagnetic theory, during the whole irradiation time of 220 seconds, thus in summary one can say in the beginning water had the maximum temperature profile followed by Kuwait oil, UAE Oil, then Saudi Arabia oil, however at end of irradiation time, Kuwait oil has the maximum thermal capacity followed by UAE oil, water then Saudi Arabian crude oil.

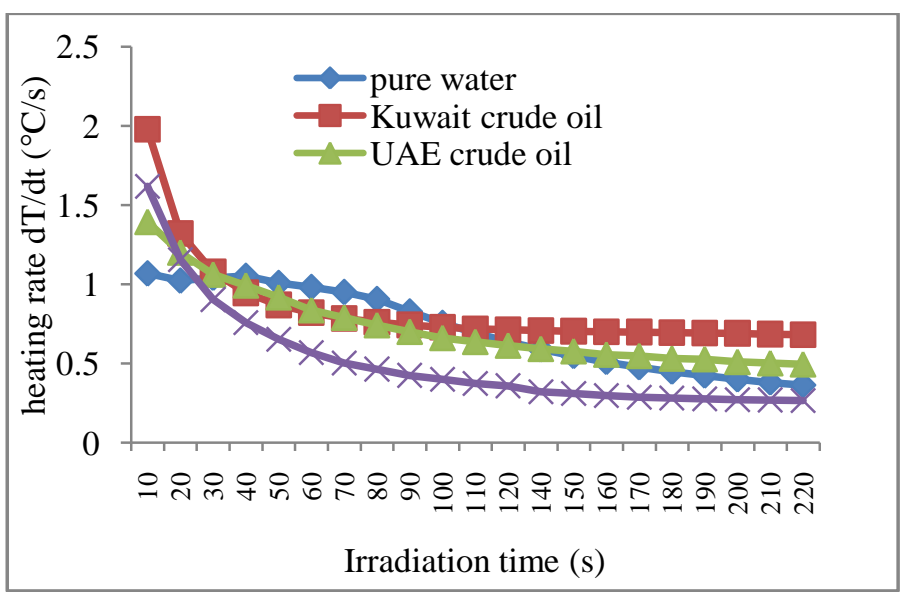

Fig 4: Heating rates of the pure components at $800 \mathrm{Watt}$ and 3.5 minutes

Figure 4 represents the heating rates of the same individual components, and shows the temperature increases with time, and that would give a clear idea on how materials would responds to microwave and the wave absorption and heat generation varies with time, since it is well known that microwave heating is energy conversion and materials absorb microwave energy by two mechanisms, one ionic conduction and the other is dipole rotation that depends strongly on the temperature of the sample, polarity of the sample as well as conductivity of the sample, hence in the current experimental observations, in contrast to what had been seen with temperature vs. time curves (figure 3), every component have different initial rate of temperature increase, although it reaches the maximum very fast, in first 30 seconds of radiation all of the curves had converged at heating rate of around $1.2^{\circ} \mathrm{C} / \mathrm{s}$ and from that convergence point as it can be seen from the figure; the heating rate of water had exceeded the others, hence the heating rate of the Kuwait and UAE oils were observed to decrease after they have converged with the water curve, regarding the Saudi Arabian crude oils the heating rate was drastically decreased in comparision to the other components.

Regarding the water again; the heating had reached a second maximum of around $1.20 \mathrm{C} / \mathrm{s}$, after which it started to decrease until it crosses the curves of both Kuwait and UAE oils which have had reached a plateau at around 0.8 and $0.5{ }^{\circ} \mathrm{C} / \mathrm{s}$ respectively but it has not reached the Saudi Arabian crude oil whose plateau was at around $0.4{ }^{\circ} \mathrm{C} / \mathrm{s}$, Generally it's clear from this experimental results that, crude oil from Kuwait had ended up having the maximum heating rate at the beginning of the process (at low temperature) and at the end of the process (at high temperature), followed by the crude oil from Saudi Arabia which had the second highest heating rate at the beginning and the lowest heating rate at medium and high temperature. UAE crude oil has medium heating rate based on this experiment at the beginning at low temperature and medium as well as at high temperature, and finally water had the lowest heating rate at low temperature and have the highest at medium temperature and medium heating rate at high temperature, however these results were not in accordance with the literature according to which water should have had the maximum heating rate over all types of oil and that is because first, water is a polar molecules with permanent dipoles and secondly it has higher dielectric properties than the non-polar hydrocarbon oils, however this contradictions may be as a result of the fact that crude oils are mixture of thousands of component including charges carrying components such as fatty acids, heavy metals (Sulfurs, vanadium, Nickel and the likes). All of these compounds may have contributed synergistically and gave the current unusual results, however most of the previous articles claimed that water would have higher heating rate than crude oils but the current experimental work showed just the opposed, however, the theory still hold for some cases, for example the case of the Saudi Arabia oil; it does have lower heating rate and also lower temperature than water.

\subsection{The Volumetric Rate of Heat Generation of the}

\section{Individual Components}

Basically, materials absorb microwave energy through by the dielectric properties, and that is the reason why some time microwave material processing is referred to energy conversion instead of heat transfer, since microwave also does not obey the common rule of conventional heating in which heat is transferred either through conduction or convection from the high temperature location to lower temperature location, thus it is important for an investigator to have some rough idea on how the heat is generated within the volume of the sample as the irradiation goes on, together with the dielectric properties of the component which are function of temperature. 


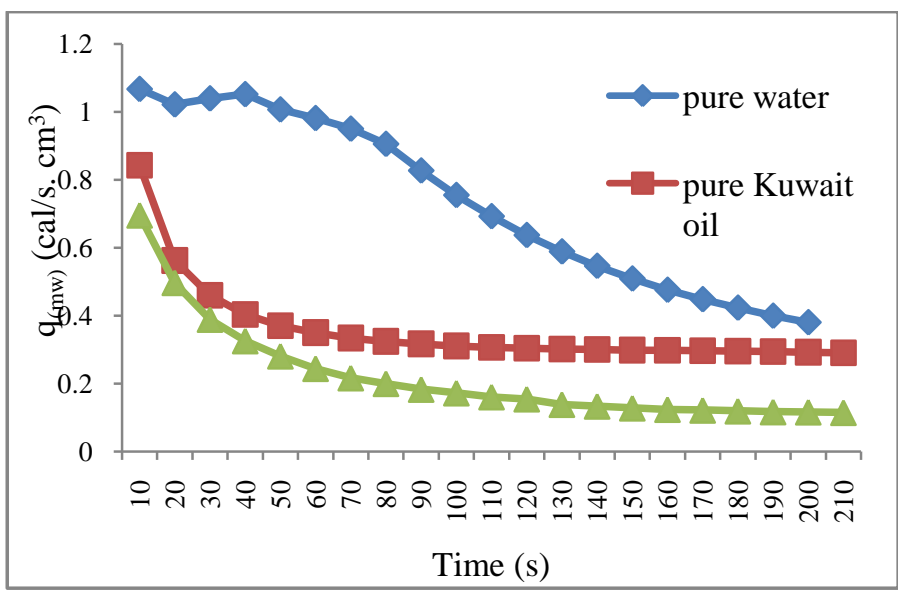

Fig 5: The volumetric rate of heat generation of the singles phases at 800 watt for 3.5 minutes

Figure 5 represents the experimental results of the volumetric rate of heat generation of different materials at their single phase states via microwave energy, the values of volumetric heat generation was calculated by inserting the temperature data in equation 3 , hence figure 5 depicted the data of the pure substance at their single phase states namely pure water, pure Kuwait oil and pure S.A. oil, at the beginning of the irradiation specifically within the first 10 seconds all materials were found to have the highest capacity to generate heat from the incident electromagnetic waves, then in the subsequent period two distinct trends were observed, in one trend the volumetric heat generation of the crude oils were found to decrease with increasing the irradiation time until they reached plateau after 60 seconds. despite that Kuwait crude oil was found to have high capability to generate more heat than Saudi Arabia oil, from the same figure; the curve of the Kuwait crude oil was started at $0.8 \mathrm{cal} / \mathrm{s} * \mathrm{~cm} 3$, and decayed as time went on till reached plateau at $0.4 \mathrm{cal} / \mathrm{g} * \mathrm{~cm} 3$, while that of the Saudi Arabia had started at around $0.7 \mathrm{cal} / \mathrm{g} * \mathrm{~cm} 3$ and reached plateau at around $0.3 \mathrm{cal} / \mathrm{g} * \mathrm{~cm} 3$ and that may be due to the elemental composition of each crude as reported earlier in the literature. Secondly; water was found to have different trend that had the highest quantitative values of the volume rate of heat generation over the crude oils this behavior is in accordance with the theory, since water possess high polarity and high dielectric properties as well, another significant information is that the highest values of heat generation in water sample were observed at intermediate irradiation time (from 30s to 100s), unlike the crude oils whose maximum heat generation was observed at the beginning then decreased as the irradiation goes on until the plateau.

\subsection{Dielectric Properties}

The upcoming Figure 6 shows the dielectric properties of same previously discussed single phase components, however the result was found to be in accordance with the theory in the sense that water had the highest dielectric constant and dielectric loss; another significant observation is that all crude oil were shown to possess very little dielectric properties and this rise some ambiguities since it has already seen previously that crude oils are generating heat out of the electromagnetic wave almost in the same fashion as water does. Moreover the temperature raise of the Kuwait crude oil had even exceeded that of the water (refer to Figure 3) this is to somehow in contradiction with the general rule of thumb of the electromagnetic

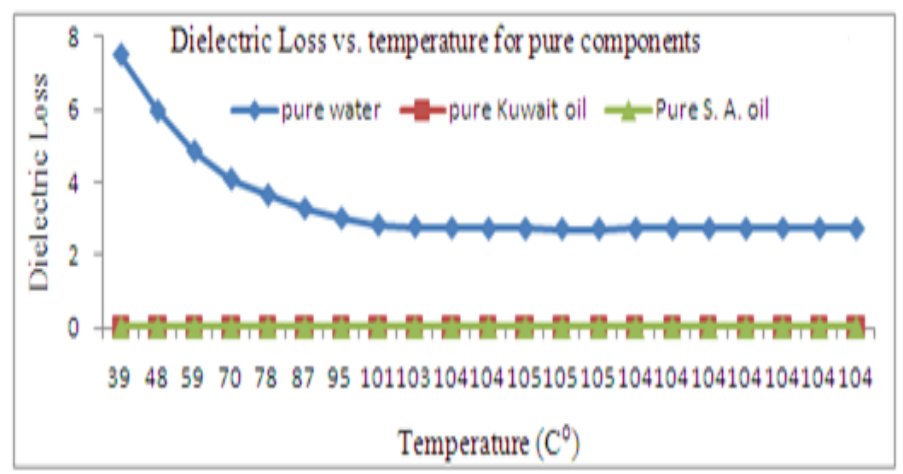

Fig 6 : Dielectric Loss of the pure components

Theory, which says only the dielectric materials can absorb the incident wave and convert them to heat, however researcher attributed this to the fact that, crude oils naturally consists of thousands of component among of which some charge carrying elements or ions that could align and rearrange or even move Within the sample as a response to the incident wave, then this movement would lead to energy generation that is evidenced by the rise in sample temperature, no much research have been done thus far on how the trace elements on the crude oil could contribute to the heating process other than the well known fact that microwave does not penetrate the opaque materials. The trend of the water sample curve is to some extend similar with finding by Li Dawei, who observed a decrease in the dielectric loss of the water contaminated soil sample with increasing temperature, when temperature reached beyond $100^{\circ} \mathrm{C}$, however he explained that because of evaporation of the water molecules in the soil sample (phase change) (Li Dawei et al., 2009).

Avijid and coworker have investigated the thermal profile of some porous metals, and postulated that electrically conducting minerals does absorb microwave power but their absorption is just limited to their skin depth which is a function of material permittivity, conductivity and angular frequency (Avijid et al., 2009).

J.P. Robinson has investigated the dielectric properties of moist soil sample, they found it to increase with increasing temperature, at temperature below 100 (J. P. Robinson et al., 2012). 


\subsection{Wavelength and Penetration Depth of the Individual Components}

This section would elaborate about the behavior of the electromagnetic wave itself when it penetrates through materials, in terms of wavelength and penetration depths.

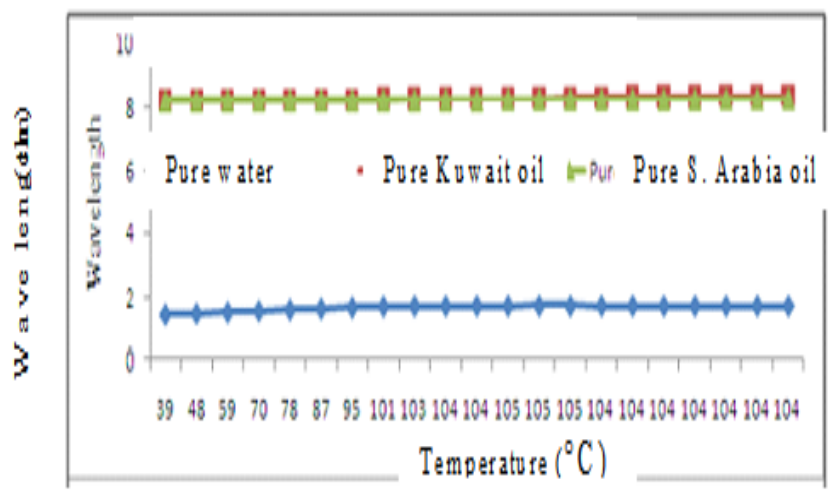

Fig 7: wave length of the pure components, $(0.05$ and $0.1 \%$ are the concentration of the demulsifiers used)

Figure 7 represents the wavelength of microwave on the pure components, from experimental data depicted in this figure it was clear that microwave had a very short wave length in water sample, hence the wave length was around $1.7 \mathrm{~cm}$ and 8 $\mathrm{cm}$ for water and crude oils respectively, this means there must be some dissipation of microwave in another word the electromagnetic waves are dissipated in the medium, absorbed and converted to energy while its passing through, and that was clearly evidenced by the increase in the sample temperature as the irradiation goes on, and that is due to the ion mobility or ionic conduction factor were mentioned in earlier section of this study. Jeffrey and friends have reported the dependencies of the dielectric properties on the temperature of the system (Jeffrey D. Macklis et al., 1979).

\section{CONCLUSIONS}

The heating pattern of water and crude oil was successfully carried out in this experimental study, from this experimental results, it was found that all materials shown to respond well to the microwave irradiation, and that was evidenced from the temperature curves in which sample temperatures were found to increase with increasing irradiation time, however, water curve had reached a plateau at $100^{\circ} \mathrm{C}$, but oil samples have not attained any critical plateau.

With regard to wavelength in the samples, water had the shortest wavelength of $2 \mathrm{Cm}$, followed by oils whose wavelengths had an identical numerical value of $8 \mathrm{Cm}$. hence this results proved that both Kuwait and Saudi Arabia crude oils respond well to microwave energy, that might be due to the heteroatoms, asphaltenes, naphthenic acids and other microwave susceptible molecules which might act as free charges promoting ion mobility and migration effects.

\section{REFERENCES}

[1]. Quan Bu et al., 2013, Renewable phenols production by catalytic microwave pyrolysis of duglas for sawdusts, J. Of, Bioresource Technology, doi: http://dx.doi.org/10.1016/j.biortech.2013.05.073.

[2]. J. P. Robinson et al., 2008., thermal desoption and pyrolysis of oil contaminated drill cutting by microwave heating, J.of Anal. Appl. Pyrolysis 81 27-32

Mingfu Huang et al., 2006, Removal of naphthenic acid by microwave, J. Of cleaner production, 14 736-739.

[3]. Vasily A. Bolotov et al., 2012, Pyrolysis of heavy hydrocarbones under the microwave heating of catalysts and adsorbents, Journal of Microwave Power and Electromagnetic Energy, 46 (1), pp. 39-46.

[4]. Adrian Porch et al., 2012, Microwave treatment in oil refining, J. Of applied petroleum resources, 2: 37-44

[5]. Abdurahman H. Nour and Rosli M. Yunus 2006, A continuous microwave heating of water-in-crude oil emulsions: An experimental study, J. of Applied science, 6 (8): 1868-1872, 2006

[6]. Jeffrey D. Macklis et al., 1979, Temperature dependance of the microwave properties of aqueous solutions of ethylene glycole between $+15^{\circ} \mathrm{C}$ and $-70^{\circ} \mathrm{C}$, J. OF Cryobiology 16, 272-286.

[7]. F. Kormin et al., 2013, Heating mechanisms of temperature controlled microwave closed system (TCMCS), International Journal of Engineering Science and Innovative Technology (IJESIT) Volume 2, Issue 5

[8]. Sateesh Mutyala et al.,2010, Microwave applications to oil sand and petroleum : A review, J. of fuel processing technology, 91 127-135.

[9]. D. A. Jones et al.,2002, Microwave heating applications in envirnmental engineering- a review, J. Of Resources concervation and recycling 34 75-90

[10]. T. J. Appleton et al., 2005, Microwave technology for energy efficient processing of waste, J. Of Applied energy 81, 85-113.

[11]. J. P. Robinson et al.,2012, Microwave remidation of hydrocarbon-contaminated soil-scal-up using batch reactor, J. Of seperation and purification technology 96 12-19.

[12]. Zhifeng $\mathrm{Hu}$ et al., 2012, A steady on experimental characteristic of microwave-assisted pyrolysis of microalgae, J. Of Bioresource technology 107 487-493.

[13]. J. Zhu et al.,2007, mathematical modeling of continuous flow microwave heating of liquids (effects of dielectric properties and design parameters), International $\mathrm{j}$. of thermal science 46 328-341.

[14]. Li Dawei et al., 2009, Microwave thermal remidation of crude oil contaminated soil enhanced by carbone fiber, J. Of envirnmental sciences, 21 1290-1295. 
[15]. N. Bjorndalen et al., 2004, the effect of wicrowave and ultrasonic irradiation on crude oil during production with a horizontal well, J. of petroleum science and engineering 43 139-150.

[16]. Su shiung lam et al., 2012, Microwave heated pyrolysis of waste automotive engine oil: influence of operation parameters on the yield, composition and fuel properties of pyrolysis oil, J. Of ful 92 327-339.

[17]. H. Shang et al.,2006, Microwave treatment of oil contaminated north sea drill cuttings in a high power multimode cavity, J. Of separation and purification technology 49 84-90

[18]. J. A. Menendez et al., 2010, Microwave heating process involving carbone materials, J. of fuel processing technology.

[19]. Gallawa, J.C. (1989). The Complete Microwave Oven Service, Handbook: Operation, Maintenance, Troubleshooting and Repair. Library of Congress Cataloging in Publication Data

[20]. J. Zhu et al., 2007, Mathematical modeling of continuous flow microwave heating of liquids (effects of dielectric properties and design parameters), International journal of thermal sciences 46 328-341.

[21]. Rocio Perez-Paes et al., 2008, Separation of oil watersludge emulsions coming from palm oil mill process through microwave techniques. International microwave power institude 42-3-39

[22]. Avijit et Mondal et al., 2009, Microwave heating of pure copper powder with varying particle size and porosity, J. Of microwave power and electromagnetic energy online 43-1

[23]. Wolf, N.O. (1986). Use of Microwave radiation in Separating Emulsions and Dispersion of Hydrocarbon and water U.S.Patent 4,582.629 